\title{
A Strategy for the Prediction of the Response of Hysteretic Systems: A Base for Capacity Assessment of Buildings under Seismic Load
}

\author{
Liborio Cavaleri*, Fabio D. Trapani, Giuseppe Macaluso and Gaia Scaduto
}

Dipartimento di Ingegneria Civile, Ambientale, Aerospaziale, dei Materiali - Università di Palermo, Palermo, Italy

\begin{abstract}
A statistical non linearization method is used to approximate systems modeled by the Bouc differential equation and excited by a Gaussian white noise external load. To this aim restricted potential models (RPM) are used, which are suitable for an extended number of nonlinear problems as have been proved several times. Since the solution of RPM is known by the probabilistic point of view, all statistical characteristics can be derived at once with advantages by the computational point of view. Hence, this paper discusses the possibility to determine sets of parameters characterizing potential models that are valid for describing a hysteretic behavior. In this way the characterization of the hysteretic behavior of a system can be performed with computational efforts lower than that normally requested.
\end{abstract}

Keyword: Bouc model, energy dissipation, equivalent non linearization, hysteretic behavior, response statistics, restricted potential models.

\section{INTRODUCTION}

The possibility of dissipation of energy under cyclic loading is basic for structures in seismic zones. This is a characteristic that contributes to prevent the crash and for this reason design strategies are commonly adopted that allow the exhibition of a structural ductile behavior. In this context a reliable assessment of the structural capacity requests a reliable modeling of the energy dissipation (viscous and hysteretic) capacity.

With regards to the behavior under cyclic excitations, solutions were found for dissipative systems based on generalized differential equations. These equations were at once applied in the case of stochastic excitations (earthquake, wind etc.).

A solution for dynamic hysteretic systems was obtained by Bouc [1]. An extension of this solution was given by Barber and Noori [2]. Further, a solution for the classical assumption of a bilinear hysteresis, which is used to describe the behavior of steel with large plastic cycles, was found by Suzuki and Minai [3] later on. However more and more applications of the Bouc model or specializations/ modifications of it can be found in the literature proving its success.

Regarding the strategies for solving motion equations with stochastic excitations, different approaches are available. One class of methods consists of finding a solution of the associated Fokker-Planck-Kolmogorov equation. On the other hand the moment closure technique can be applied. Also, linearization and averaging methods can be used as in [4] and in [5] where the cases of nonlinear systems and hysteretic systems are respectively discussed. Alternatively

*Address correspondence to this author at the Dipartimento di Ingegneria Civile, Ambientale, Aerospaziale, dei Materiali - Università di Palermo, Palermo, Italy; Tel: +39 09123896733: Fax: +39 091427121;

E-mail: liborio.cavaleri@unipa.it an equivalent non linearization method may be used (e.g. [6$8]$ ), that is the substitution of the original model with an equivalent nonlinear one having characteristics that simplify the structural analysis. Referring to the modeling of hysteresis, approaches different from the Bouc model can be found in the literature (e.g. [9-11]).

In the field of equivalent non linearization, the main purpose here is to check if differential nonlinear equations exist, whose solution is known in a symbolic form by the probabilistic point of view, capable to describe the hysteretic behavior of a Bouc system. Since for the class of restricted potential models discussed in $[7,8]$ it is known that the exact expression of the probability density function of the processes that governs, and the suitability of these models to represent the behavior of a large number of typologies of systems (e.g. [12-16]) has been proved, in this paper this class of equations will be investigated. Hence in this paper, first the characteristics of Bouc models and potential models will be presented, then an equivalent non linearization technique will be described and specialized in two different methods involving only the dissipation forces. Also, applications will be presented in order to verify the degree of reliability of any equivalent model. Finally a modification of the presented equivalent non linearization approaches will be discussed involving the dissipation forces and the restoring forces.

\section{STOCHASTIC EQUIVALENT NON LINEARIZA- TION OF HYSTERETIC SYSTEMS BY POTENTIAL MODELS}

The hysteretic systems described by Bouc [1] can be modeled by the following equation

$$
\ddot{X}(t)+c \dot{X}(t)+k_{x} X(t)+k_{z} Z(t)=W(t)
$$

where $\ddot{X}(t), \dot{X}(t), X(t)$ mean respectively acceleration, velocity and displacement and depend on time $t, c$ is the parameter describing the viscous damping, $k_{x}$ and $k_{z}$, 
properly combined, condition the stiffness in the elastic stage and in the post elastic stage, $W(t)$ is a Gaussian zero mean white noise process acting as an external force on the system, finally $Z(t)$ is an additional state variable described in the generalized differential equation:

$$
\dot{Z}=A \dot{X}-\gamma|\dot{X}| Z\left|Z^{n-1}\right|-v \dot{X}\left|Z^{n}\right|
$$

For simplicity the system is normalized in such a way that the dynamic mass is set to one.

Because of the computational effort in finding the solution of Eq. (1) in terms of probability density function of the state variables (Monte Carlo simulation) and because of the extreme approximation of the approaches based on equivalent linearization or on a moment closure, an equivalent non linearization is discussed here based on the use of Restricted Potential Models (RPM) [7, 8] whose equation is:

$$
\ddot{X}+\frac{d Q(H)}{d H} \dot{X}+r(X)=W(t) ;
$$

The displacements of the system, as in the case of Eq.(1), are described by the process $X(t)$ depending on time $t$. $W(t)$ is a Gaussian zero mean white noise process acting as an external force on the system, while $H$ is the total energy of the system, that is

$$
H(X, \dot{X})=\frac{1}{2} \dot{X}(t)^{2}+\int_{y=0}^{X(t)} r(y) d y
$$

where $r$ is the restoring force generally expressed as a nonlinear function of the displacement $X$. Finally, the damping "coefficient" is a derivative of a function $Q$, depending on the total energy $H$ of the system.

The solution of the Fokker-Planck-Kolmogorov equation associated to RPM is known. This is an important advantage of this class of models. This solution is the joint probability density function of the displacement and the velocity, $p_{x, \dot{x}}(x, \dot{x})$, given in the form

$$
p_{x, \dot{x}}(x, \dot{x})=q \exp \left(-\frac{1}{\pi S_{0}} Q(H(x, \dot{x}))\right)
$$

where the symbol $S_{0}$ refers to the intensity of the white noise process $W(t)$ with the relationship

$$
\begin{aligned}
& 2 \pi S_{0} \delta\left(t_{2}-t_{1}\right)=E\left[W\left(t_{1}\right) W\left(t_{2}\right)\right] \\
& \delta\left(t_{2}-t_{1}\right)
\end{aligned}
$$

being the Dirac delta while $\mathrm{E}[\cdot]$ is the average operator.

The parameter $q$ is a normalization factor given by

$$
\frac{1}{q}=\int_{-\infty}^{\infty} \int_{-\infty}^{\infty} \exp \left(-\frac{1}{\pi S_{0}} Q(H(x, \dot{x}))\right) d x d \dot{x} .
$$

Hence, potential models have a known solution by the probabilistic point of view. Therefore it may be useful to approximate a hysteretic system which is described in Eqs.(1-2) by a potential model described in Eq.(3) with the solution given in Eq.(5).

The equivalent non linearization technique is very similar to the equivalent linearization one, the only difference is that, in the first case, the substituting system itself is a nonlinear system.
If the restoring force in Eq.(3) is chosen as

$$
r(X)=k_{x} X
$$

the total energy becomes

$$
H(X, \dot{X})=\frac{1}{2} \dot{X}^{2}+\frac{1}{2} k_{x} X^{2} .
$$

The difference $\varepsilon$ between the two systems described in Eq.(1) and in Eq.(3) is given by

$$
\varepsilon=\frac{d Q(H)}{d H} \dot{X}-c \dot{X}-k_{z} Z
$$

Further, if the function $Q$ is chosen as a polynomial function of the total energy $H$, that is

$$
Q(H)=\sum_{i=1}^{k} a_{i} H^{i}
$$

the joint probability density function becomes

$$
p_{x, \dot{x}}(x, \dot{x})=q \exp \left(-\frac{1}{\pi S_{0}} \sum_{i=1}^{k} a_{i}\left(\frac{1}{2} \dot{x}^{2}+\frac{1}{2} k_{x} x^{2}\right)^{i}\right)
$$

with $q$ reducing to

$$
\frac{1}{q}=\int_{-\infty}^{\infty} \int_{-\infty}^{\infty} \exp \left(-\frac{1}{\pi S_{0}} \sum_{i=1}^{k} a_{i}\left(\frac{1}{2} \dot{x}^{2}+\frac{1}{2} k_{x} x^{2}\right)^{i}\right) d x \cdot d \dot{x}
$$

and the error $\varepsilon$ becomes

$$
\varepsilon=\sum_{i=1}^{k} i a_{i}\left(\frac{1}{2} \dot{X}^{2}+\frac{1}{2} k_{x} X^{2}\right)^{i-1} \dot{X}-c \dot{X}-k_{z} Z .
$$

The minimization of the averaged quadratic error with respect to the parameters $a_{i}$

$$
E\left[\varepsilon^{2}(X, \dot{X}, Z)\right] \rightarrow \min _{a_{i}},
$$

can be done setting the gradients to zero:

$$
\frac{\partial}{\partial a_{i}} E\left[\varepsilon^{2}(X, \dot{X}, Z)\right]=0, i=1 \ldots . . k .
$$

The derivatives are sorted in the linear system

$$
\mathbf{A} \cdot \mathbf{a}=\mathbf{d},
$$

where $\mathbf{a}$ is a vector of the parameters $a_{i}$ and $\boldsymbol{A}$ and $\boldsymbol{d}$ respectively are a matrix and a vector of averages:

$$
\boldsymbol{A}=\left[\begin{array}{cccc}
E[\dot{X}] & 2 E\left[H \dot{X}^{2}\right] & \ldots & k E\left[H^{k-1} \dot{X}^{2}\right] \\
2 E\left[H \dot{X}^{2}\right] & 4 E\left[H^{2} \dot{X}^{2}\right] & \ldots & 2 k E\left[H^{2} \dot{X}^{2}\right] \\
\cdot & \cdot & \ddots & \\
\cdot & \cdot & \ddots & \cdots \\
k E\left[H^{k-1} \dot{X}^{2}\right] & 2 k E\left[H^{k} \dot{X}^{2}\right] & \ldots & k^{2} E\left[H^{2(k-1)} \dot{X}^{2}\right]
\end{array}\right],
$$




$$
\boldsymbol{a}=\left[\begin{array}{c}
a_{1} \\
a_{2} \\
\cdot \\
\cdot \\
a_{k}
\end{array}\right], \boldsymbol{d}=\left[\begin{array}{c}
E[M] \\
2 E[H M] \\
\cdot \\
\cdot \\
\cdot \\
k E\left[H^{k-1} M\right]
\end{array}\right]
$$

with the abbreviation

$$
M=c \dot{X}+k_{z} Z \dot{X}
$$

The moments in the vector $\boldsymbol{d}$ depend on the hysteretic state variable $Z$ defining the Bouc model. Solving Eq.(17) with respect to the vector $\boldsymbol{a}$ allows the identification of the potential model equivalent to the hysteretic system.

\section{CRITERIA FOR SOLVING EQ. (17)}

For solving Eq.(17) the evaluation of the moments contained in the vector $\boldsymbol{d}$ needs. Because the substituting potential model does not depend on the hysteretic variable $Z$, Monte-Carlo simulations by the nonlinear Bouc system have to be used to evaluate these moments. In the further calculations, these moments are fixed. The moments of the matrix A can be obtained from the Monte-Carlo simulation (method 1) or deriving the moments from the joint probability density function of the potential model (method 2) as better explained below. In both cases, considering the symmetry of the matrix $\mathbf{A}, k(k+1) / 2$ moments have to be evaluated, that is

$$
\begin{gathered}
A_{i j}=i \cdot j \cdot E\left[\left(\frac{1}{2} \dot{X}^{2}+\frac{1}{2} k_{x} \dot{X}^{2}\right)^{i+j-2} \dot{X}^{2}\right] \\
1<i<k ; 1<j<k ; j \geq i
\end{gathered}
$$

After the determination of the parameters $a_{i}$ of the equivalent potential model, for a comparison between the Bouc model and the equivalent potential model, the hysteretic state variable $\mathrm{Z}$ can be obtained assuming that the error $\varepsilon$ of the Eq.(14) is almost zero, that is

$$
Z=\frac{1}{k_{z}}\left[\sum_{i=1}^{k} i a_{i}\left(\frac{1}{2} \dot{X}^{2}+\frac{1}{2} k_{x} X^{2}\right)^{i-1} \dot{X}-c \dot{X}\right],
$$

\subsection{Method 1}

In this case the moments appearing in the matrix $\boldsymbol{A}$ depend on the variables $X$ and $\dot{X}$ while the moments appearing in the vector $\boldsymbol{d}$, considering Eq.(19), depend on the variables $X, \dot{X}$ and $Z$. All these moments can be calculated by a Monte Carlo simulation carried out by using the Bouc model. Once the above moments are calculated the unknown $\boldsymbol{a}$ of Eq.(17) can be obtained. In conclusion the method 1 for obtaining the equivalent potential model includes the following steps:

1) Fixing the number $k$ of the parameters, $a_{i}$, to be used for defining the dissipation force of the equivalent potential model;

2) Calculating the $k x k$ entries of the matrix $\boldsymbol{A}$ and the $k$ entries of the vector $\boldsymbol{d}$ by a Monte Carlo simulation by using the Bouc model;
3) Solving Eq.(17) and obtaining the $k$ coefficients defining the dissipative force of the equivalent potential model;

4) Specializing Eq.(12) for any stochastic analysis of the Bouc system.

\subsection{Method 2}

Considering that the moments appearing in the matrix $\boldsymbol{A}$ depend on the variables $X$ and $\dot{X}$, the entries of the matrix $\boldsymbol{A}$ can be calculated in dependency of the distribution of the substituting potential model. This approach needs for a double integration in fact the moment $E[f(X, \dot{X})]$ of the generic function $f(X, \dot{X})$ of the response is obtained as

$$
E[f(X, \dot{X})]=\int_{-\infty}^{\infty} \int_{-\infty}^{\infty} f(X, \dot{X}) p_{x, \dot{x}}(x, \dot{x}) d x d \dot{x}
$$

But by considering some simplifications, if the matrix A contains $k x k$ entries, only $k$ one dimensional integrals have to be performed.

First, reduction of the computational effort is possible for the case of a linear restoring force being true the following relation

$$
E\left[H^{i} \dot{X}^{2}\right]=E\left[H^{1+i}\right] .
$$

Further, as proved in [7], the following relation holds:

$$
E\left(H^{i}\right)=q \int_{0}^{\infty} h^{i} e^{-\frac{1}{\pi S_{0}} Q(h)} d h ; i=1, k-1 .
$$

being $q$

$$
\frac{1}{q}=\int_{0}^{\infty} e^{-\frac{1}{\pi S_{0}} Q(h)} d h
$$

The semi-infinite integrals in Eqs.(24-25) have to be solved numerically. Finally, moments of higher order can be derived using the recursion [7]

$$
\begin{aligned}
& E=\left[H^{i+k-1}\right]=\frac{1}{k a_{k}}\left\{i \pi S_{0} E\left[H^{i-1}\right]-\sum_{j=1}^{k-1} j a_{j} E\left[H^{i+j-1}\right]\right\} ; \\
& i=1, k-1 .
\end{aligned}
$$

The use of Eq.(24) and Eq.(26) depends on the unknown coefficients $a_{i}$ defining the function $Q(H)$. For this reason the evaluation of the entries of the matrix $\boldsymbol{A}$ requests an iterative procedure considering that the moments appearing in the vector $\boldsymbol{d}$, taking Eq.(19) into account, depend on the variables $X, \dot{X}$ and $Z$, these moments have to be calculated by a Monte Carlo simulation by using the Bouc model.

In conclusion the method 2 for obtaining the equivalent potential model includes the following steps:

1) Fixing the number $k$ of the parameters, $a_{i}$, to be used for defining the dissipation force of the equivalent potential model;

2) Fix arbitrarily the values of the parameters $a_{i}$; 
3) Calculating the $k x k$ entries of the matrix $\boldsymbol{A}$ by using Eqs. (23-26) and the $k$ entries of the vector $\boldsymbol{d}$ by a Monte Carlo simulation by using the Bouc model;

4) Solving Eq.(17) and obtaining the $k$ coefficients defining the dissipative force of the equivalent potential model (generally the values calculated will be different from the values assigned at the step 2);

5) Going back to the step 3 for an iterative estimation of the parameters $a_{i}$ until the difference between the values calculated at the previous iteration and the actual iteration is acceptable;

6) Specializing Eq.(12) for any stochastic analysis of the Bouc system.

\section{APPLICATION OF THE PROPOSED APPROACH}

The Bouc model shall be evaluated using a Monte Carlo simulation.

More than one realization needed to obtain stable moments for the non linearization technique. Especially higher moments were very sensitive and an average of long time series needed for this reason. Furthermore, to be able to receive a stationary response, in the application a value sufficiently far from zero was assigned to the parameter $k_{x}$, in fact assigning values close to zero, as a consequence of the numerical integration method, can produce non stationary responses.

One example shall illustrate the method. In the original Bouc model the damping coefficient was chosen to be 0.1 and the stiffness was assumed to be 1 . The value of spectral density of the input was assumed constant and equal to $S_{0}=\frac{1}{2 \pi}$. The hysteretic parameters of the Bouc model were set to $A=1, \gamma=0.9, v=0.1, \mathrm{n}=1$.

In the potential model the expansion of $H$ was at the third degree for two reasons. The first approach that was used showed that the forth term of the expansion tend to a very small negative number, therefore the influence of the parameter $a_{4}$ will be small for normal regions, but of course the probability distribution will tend to infinity at the boundaries. The other reason is a numerical problem of the second approach (method 2), namely the numbers tend to the maximum allowed dimensions for a representation with double precision.

Referring to the first method all needed moments appearing in Eq.(18) derive from the Monte-Carlo simulation. Therefore the matrix $\boldsymbol{A}$ can be solved at once and no iteration is needed. The second method evaluates the moments of matrix $\boldsymbol{A}$ from the equivalent potential model. Since these moments depend on the unknown parameters of potential model, for the estimation of the entries of the parameters $a_{i}$ an iterative procedure needs.

The parameters that were evaluated are listed in Table $\mathbf{1}$.

Each parameter has a different dimension $\left(\mathrm{a}_{1}[1 / \mathrm{s}]\right.$, $\left.\mathrm{a}_{2}\left[\mathrm{~s} / \mathrm{cm}^{2}\right], \quad \mathrm{a}_{3}\left[\mathrm{~s} / \mathrm{cm}^{4}\right]\right)$ allowing to obtain globally the dimension of an acceleration for the second term in the left side of Eq.(3). One can observe that the sets of parameters obtained by the method 1 and by the method 2 are different as a consequence of the different procedures but these two set of parameters lead to very close responses as it is proved by the comparisons in terms of response histories, dissipated energy, probability density functions, commented below. In spite of the similar results, the two methods request different computational efforts allowing method 2 to obtain a solution more quickly because of the simplified way of obtaining the terms of matrix $\boldsymbol{A}$ in Eq.(17).

Table 1. Parameters of the equivalent restricted potential model.

\begin{tabular}{|c|c|c|}
\hline & Method 1 (k=3) & Method 2 (k=3) \\
\hline \hline $\mathrm{a}_{1}$ & $2.86688 \mathrm{e}-1$ & $6.3544 \mathrm{e}-1$ \\
\hline $\mathrm{a}_{2}$ & $-5.32726 \mathrm{e}-3$ & $-1.0775 \mathrm{e}-1$ \\
\hline $\mathrm{a}_{3}$ & $1.00443 \mathrm{e}-4$ & $7.5067 \mathrm{e}-3$ \\
\hline
\end{tabular}

The results are used to compare the original system and the two approximations with an expansion up to the third degree $(\mathrm{k}=3)$. Fig. (1) presents the displacement $X(t)$, while Fig. (2) shows the velocity $\dot{X}(t)$ and the plastic parameter $Z(t)$ is shown in Fig. (3). Finally in Fig. (4) a comparison of the dissipated energies is shown.

Referring to the approach 2, at some points the hysteretic force of method 2 is outside the assumed range. This seems to be a numerical problem of the derivation. It can be seen that the derivation of the velocity results in an extending roughness. It is also obvious that a load level was chosen with great plastic reactions, since the plastic variable $Z$ keeps within the region $[-1,1]$, while the total displacement $X$ tends from $[-5,5]$.

The hysteretic behavior derived from the Bouc model and the potential model is different although the plastic force is tending to the same level. This is clear from the Figs. (56).

The joint probability distributions in accordance with Eq.(3) of the equivalent potential model are presented in Figs. (7, 8). While in Fig. (7) the results of the approach 1 are presented in the two cases of expansion $Q(H)$ defined respectively by three and four parameters, in Fig. (8) the result of the approach 2 is presented in the case of expansion $Q(H)$ defined by three parameters. A decisive difference between the probability distributions presented in the ranges represented for the state variable cannot be observed. This fact suggests to proceed in general with an expansion characterized by three parameters.

\section{RELIABILITY OF THE ASSUMED APPROXI- MATION}

The dissipative behavior of the potential model is described by the function $Q(H)$. The behavior of some of the terms of the series expansion used for $Q(H)$ is investigated in detail in this section.

If the series expansion is truncated after the forth expansion the dissipative forces of the potential system consists of nine nonlinear additive terms and one further linear, that is: 


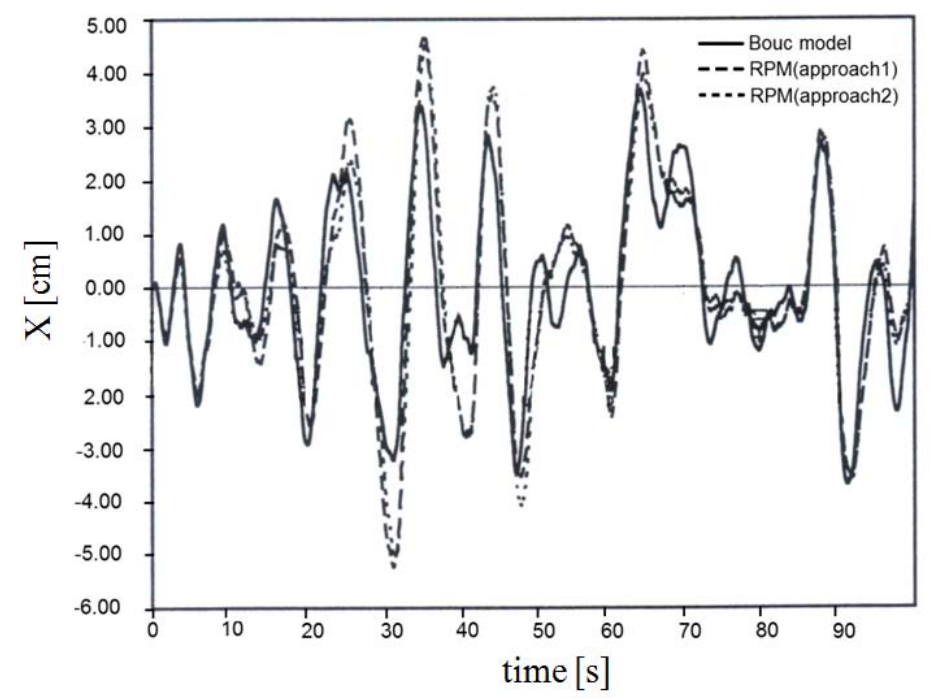

Fig. (1). Displacement history $\mathrm{X}(\mathrm{t})$ of the Bouc model and the equivalent potential model.

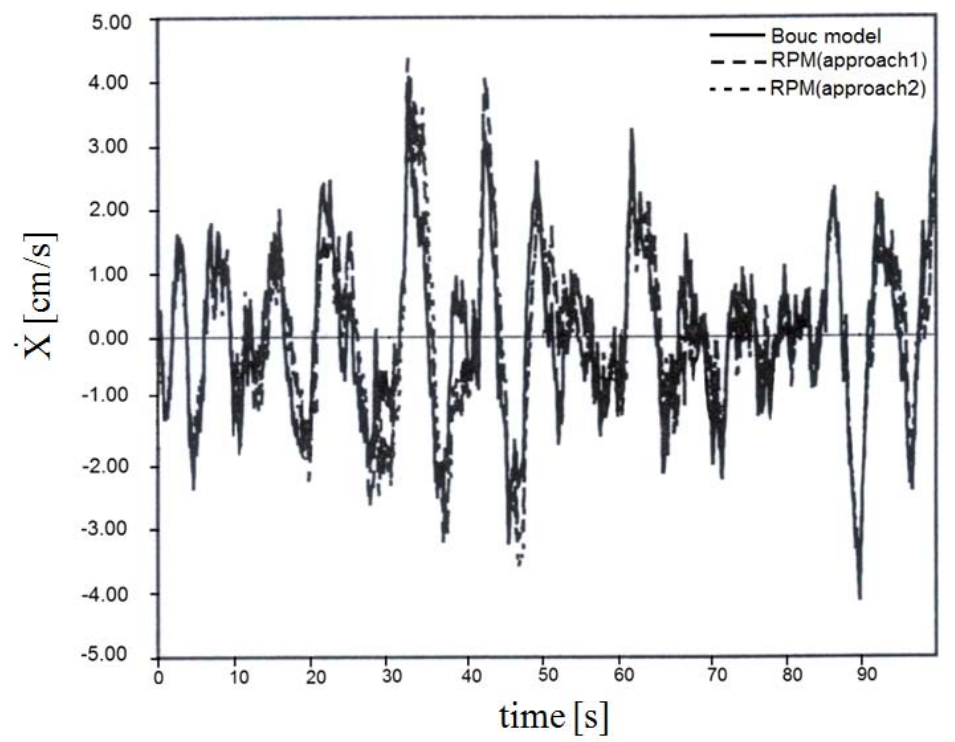

Fig. (2). Velocity history $\dot{\mathrm{X}}(\mathrm{t})$ of the Bouc model and the equivalent potential model.

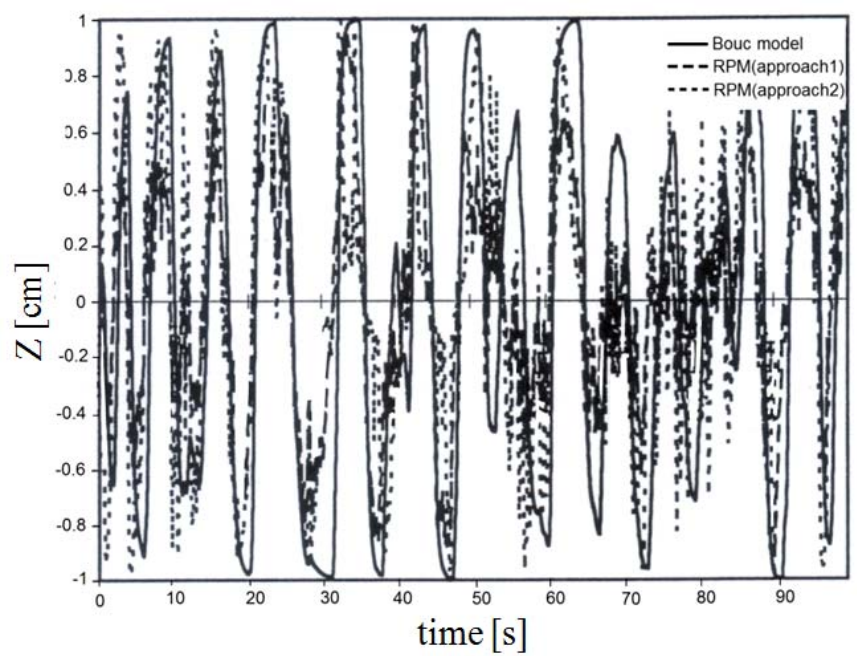

Fig. (3). Hysteretic variable history Z(t) by the Bouc model and Eq.(21). 


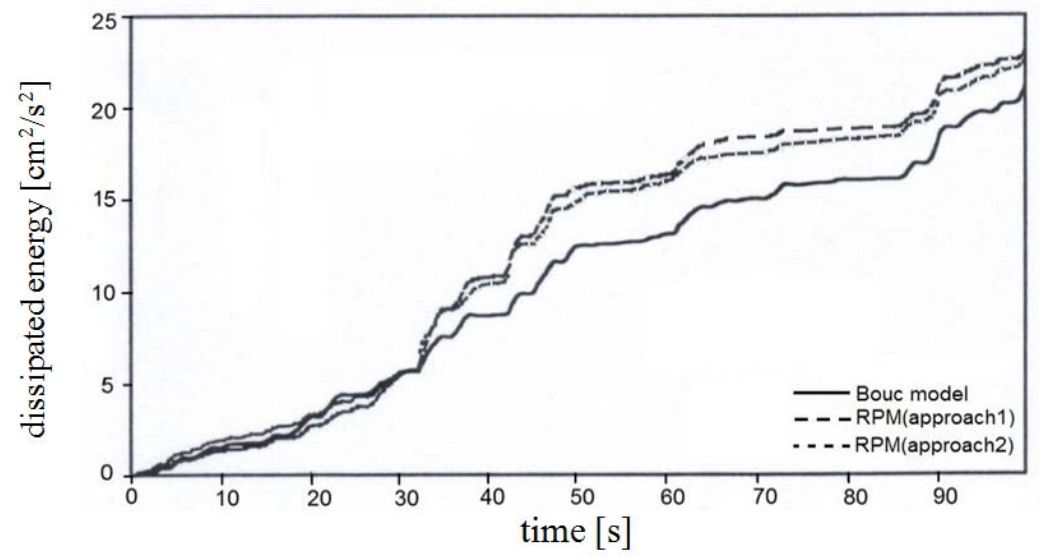

Fig. (4). Dissipated energy $\int_{0}^{t_{0}}\left(c \dot{x}(t)+k_{z} z(t)\right) \dot{x}(t) d t$ by the Bouc model and by the equivalent potential model.

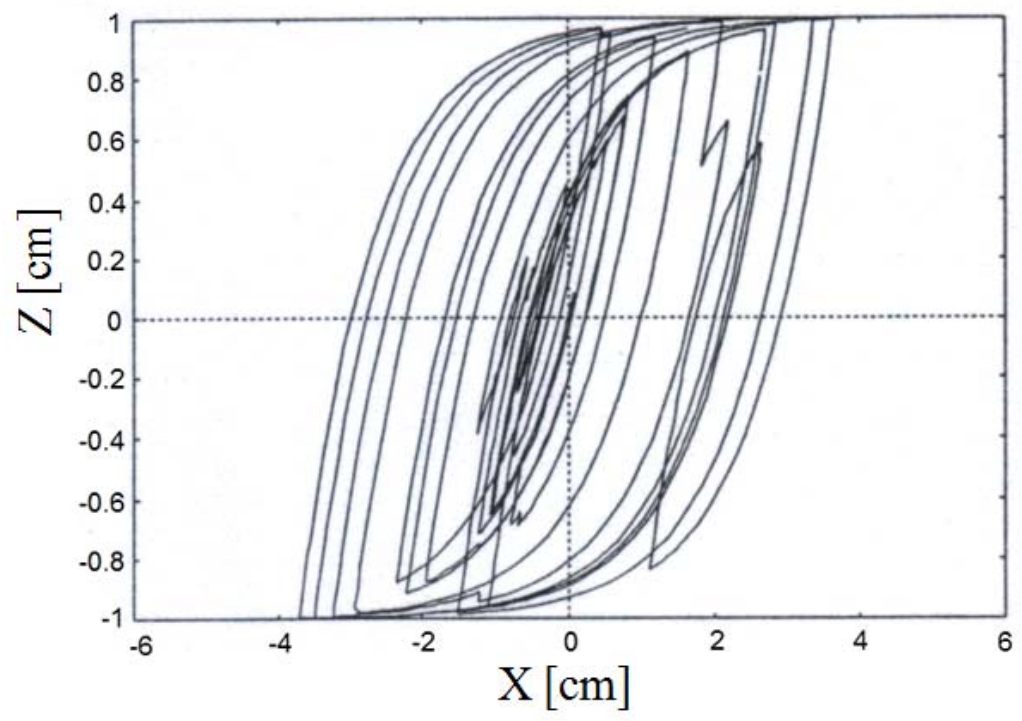

Fig. (5). Phase plot in the X-Z plane for the Bouc model.

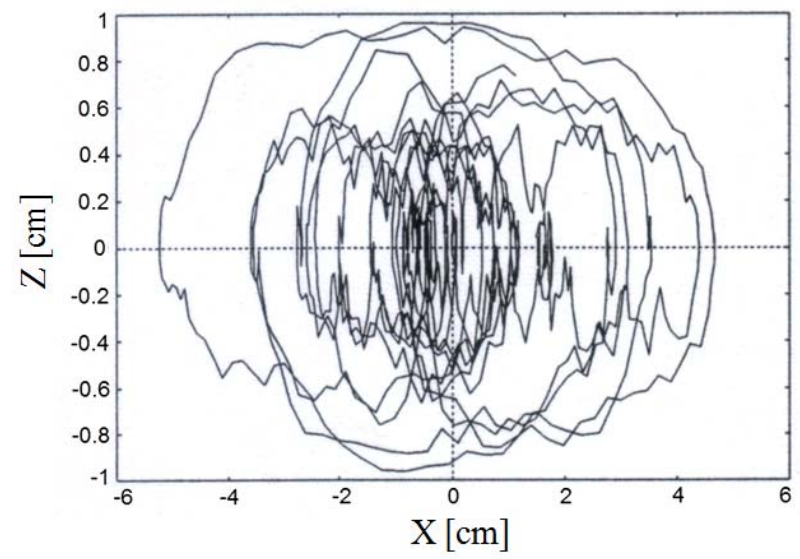

a)

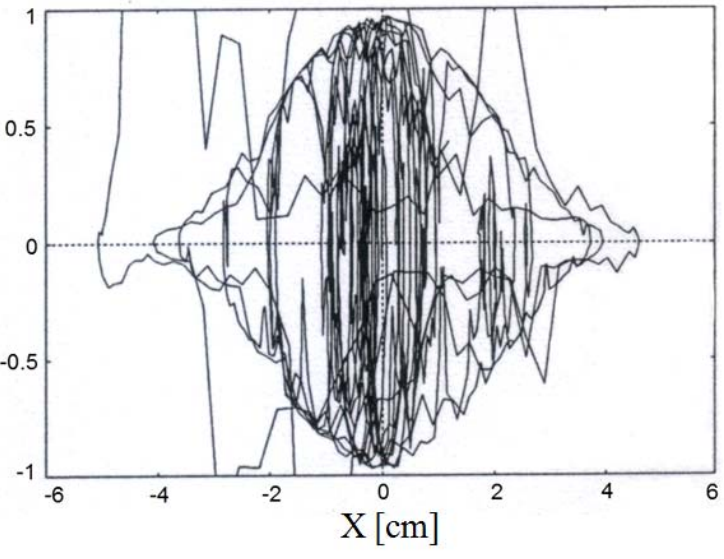

b)

Fig. (6). Phase plot in the X-Z plane for the potential model: a) method $1(k=3)$; b) method $2(k=3)$. 


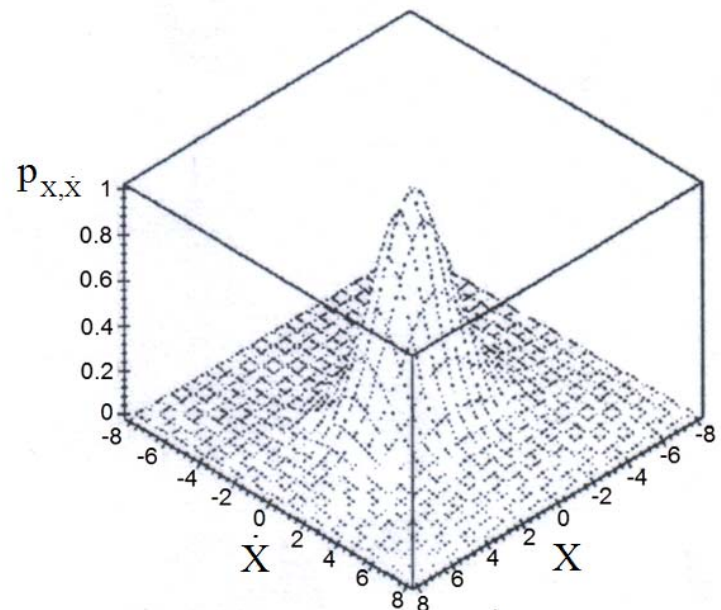

a)

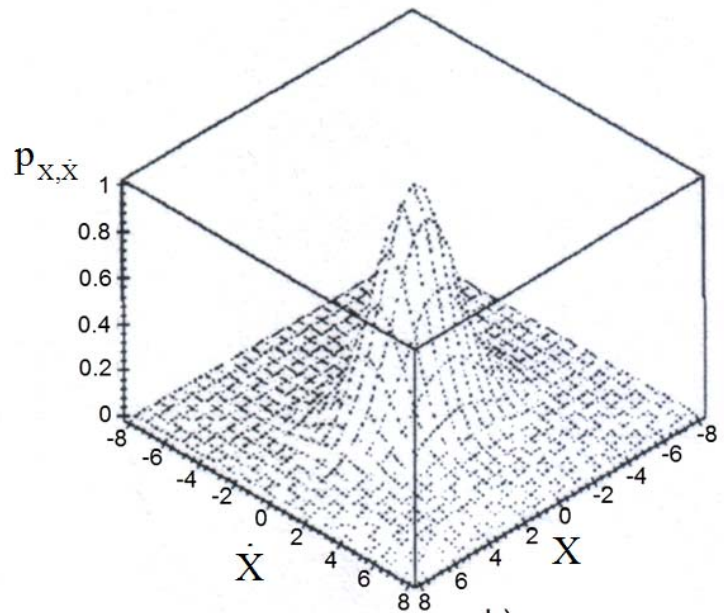

b)

Fig. (7). Joint probability distributions (not scaled by q) of the potential model: a) method $1(k=4)$; $b)$ method $1(k=3)$.

$$
\begin{aligned}
& \frac{\partial Q(H) \dot{X}}{\partial H}=a_{1} \dot{X}+a_{2}\left(k_{x} X^{2} \dot{X}+\dot{X}^{3}\right)+ \\
& a_{3}\left(\frac{3}{4} k_{x}^{2} X^{4} \dot{X}+\frac{3}{2} k_{x} X^{2} \dot{X}^{3}+\frac{4}{3} \dot{X}^{5} \dot{X}^{5}\right)+ \\
& +a_{4}\left(\frac{1}{2} k_{x}^{3} X^{6} \dot{X}+\frac{3}{2} k_{x}^{2} X^{4} \dot{X}+\frac{3}{2} k_{x} X^{2} \dot{X}^{5}+\frac{1}{2} \dot{X}^{5}\right)
\end{aligned}
$$

The sign of the dissipative force is controlled by the sign of the velocity, since all other terms are even. Therefore each singular term in Eq.(26) is not capable of representing hysteretic behavior.

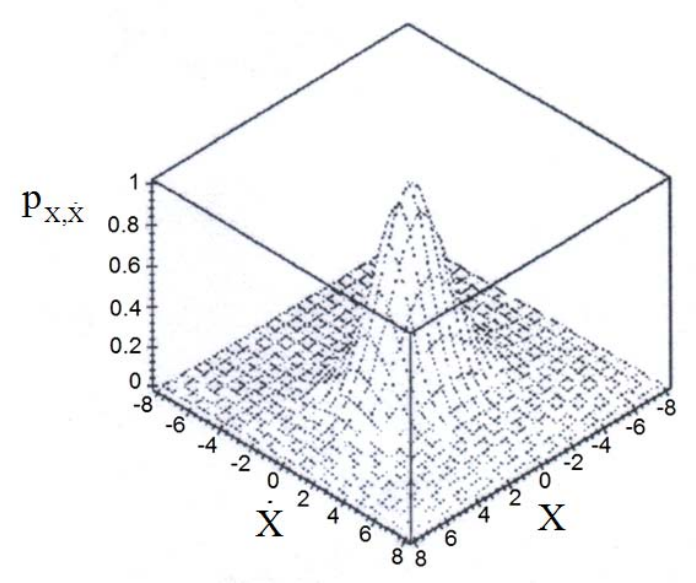

Fig. (8). Joint probability distribution (not scaled by q) of the potential model method $2(\mathrm{k}=3)$.

Since the term depending on $a_{l}$ is only a linear damping term the parameter $a_{l}$ itself can be set equal to the parameter $c$ of Eq.(6) in order to have the same linear damping as in the Bouc model. With this restriction only parameters $a_{2}$ up to $a_{4}$ can be optimized. In Figs. (9-12) the loops of the terms in Eq.(26) are presented varying the displacement cyclically. Fig. (9) is concerned with the linear damping belonging to coefficient $a_{l}$. In the same manner Figs. (10-12) present the dissipative terms belonging to $a_{2}, a_{3}$ and $a_{4}$.

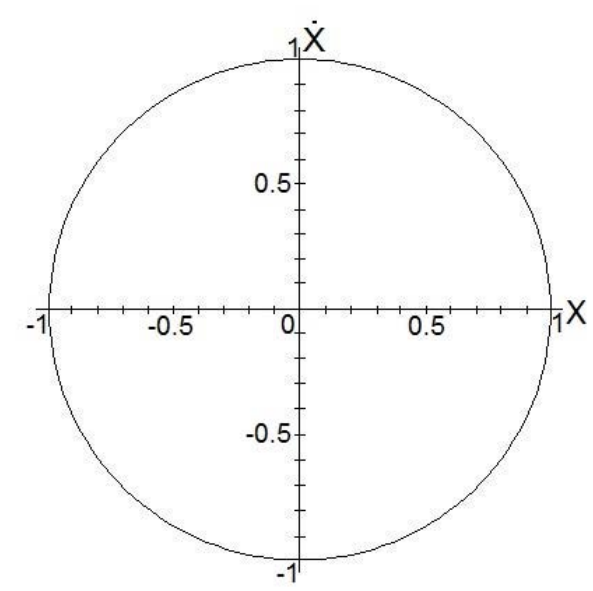

Fig. (9). Hysteresis loop for $\dot{X}$ during a periodic movement $\mathrm{X}=\sin (\mathrm{t})$.

One difference, that cannot be changed, is that at the time of an extreme value for the displacement, all nonlinear terms are zero, because at this time the velocity of the system becomes zero.

Most of the components are also zero at the zero point of the displacement. The only exception regards the factors $\dot{X}^{k}$. This is the consequence of the transforming of the hysteresis dissipated energy in damping dissipated energy.

Using Bouc model the behavior of the variable $Z$, that is proportional to the force of the hysteric part due to the displacement $X$, can be plotted. Using the potential model, $Z$ is approximated by Eq. (21). This equation depends directly on a combination of the terms presented above. As a result, at the specific points of zero velocity for the potential model the restoring force consists of $r(x)$. This is not true for the Bouc hysteresis, because in this case nonlinear restoring force has its maximum at the points of zero velocity, where the system changes its direction of movement. 

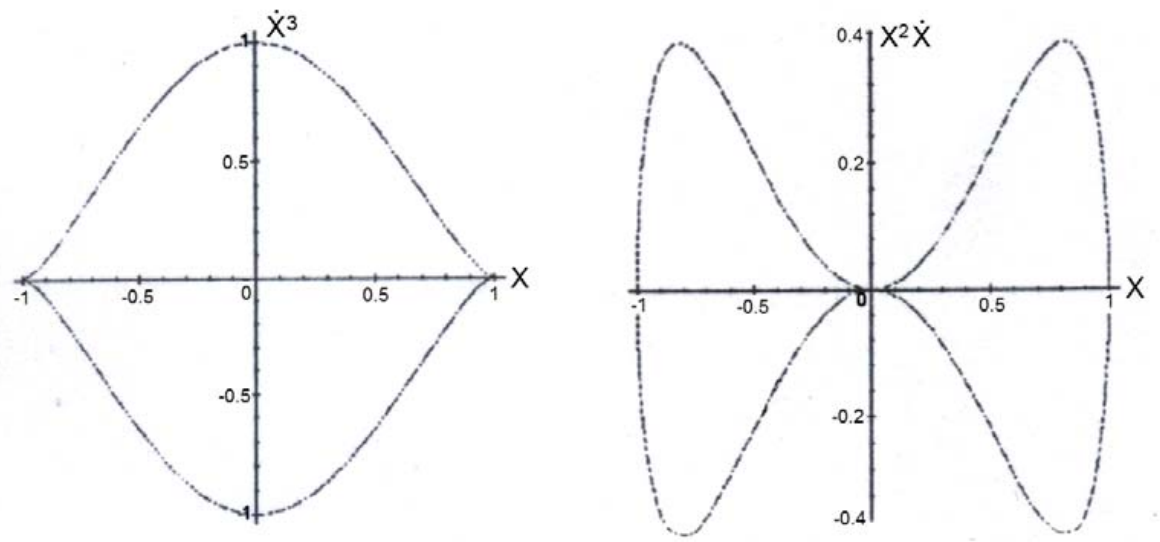

Fig. (10). Hysteresis loop of expression $\dot{X}^{2}$ and $X^{2} \dot{X}$ during a periodic movement $X=\sin (t)$.
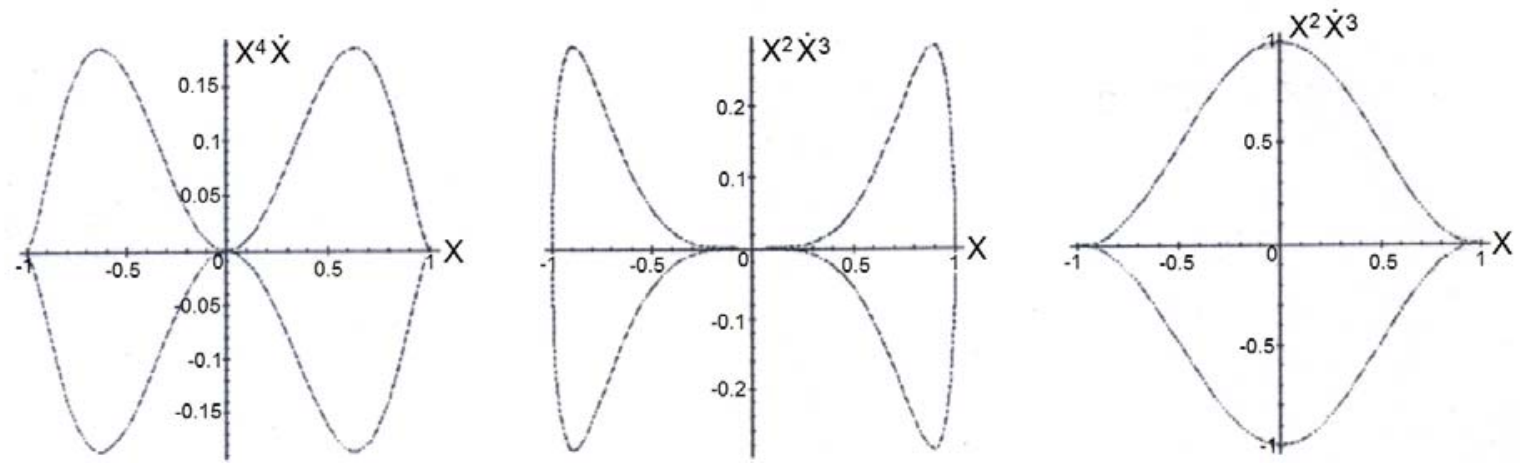

Fig. (11). Hysteresis loop of expression $X^{4} \dot{X}, X^{2} \dot{X}^{3}$ and $\dot{X}^{5}$ during a periodic movement $X=\sin (t)$.
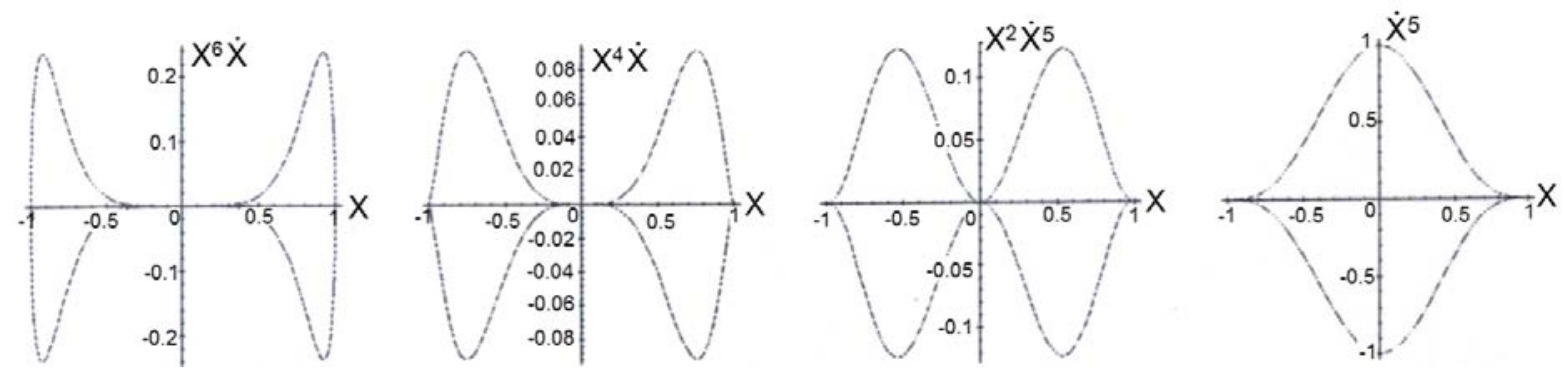

Fig. (12). Hysteresis loop of expression $X^{6} \dot{X}, X^{4} \dot{X}, X^{2} \dot{X}^{5}$ and $\dot{X}^{5}$ during a periodic movement $X=\sin (t)$.

In order to solve this problem two steps are possible. First if one does not equate the linear restoring forces of both systems the parameters on which the linear restoring forces depend can become part of the optimization process. Because of the dependency between the restoring force and the total energy the approximation can no more be separated in the way it is done at Eq. (16). In another step, the restoring terms themselves can be assumed to be parameters for the approximation process, but on the other side these parameters are coupled in products of higher order. Assuming a nonlinear symmetric behavior of the restoring force $r(x)$, for which the underlying method is valid, that is:

$$
r(X)=\sum_{j=1}^{i} b_{j}|X|^{j} \operatorname{sgn}(X),
$$

the total energy will be

$$
H=\frac{1}{2} \dot{X}^{2}+\sum_{j}^{i} \frac{b_{j}}{j+1}\left|X^{j+1}\right| .
$$

Furthermore the expression of $Q(H)$ will be

$$
Q(H)=\sum_{i=1}^{k} a_{i}\left(\frac{1}{2} \dot{X}^{2}+\sum_{j=1}^{i} \frac{b_{j}}{j+1}\left|X^{j+1}\right|\right)^{i},
$$

and the derivation with respect to $\mathrm{H}$

$$
\frac{d Q(H)}{d H}=\sum_{i=1}^{k} i a_{i}\left(\frac{1}{2} \dot{X}^{2}+\sum_{J-1}^{i} \frac{b_{j}}{j+1}|X|^{J+1}\right)^{i-1} .
$$

The coupling of the parameters $a_{i}$ and $b_{j}$ is obvious. At least the optimization has to be done minimizing the error including the additional restoring forces. 
Now the error can be expressed as:

$$
\begin{aligned}
\varepsilon= & {\left[\sum_{i=1}^{k} i a_{i}\left(\frac{1}{2} \dot{X}^{2}+\sum_{j=1}^{i} \frac{b_{j}}{j+1}\left|X^{j+1}\right|\right)^{i-1}\right] . } \\
& \dot{X}+\sum_{j=1}^{l} b_{j}|X|^{j} \operatorname{sgn}(X)-c \dot{X}-k_{x} X-k_{z} Z
\end{aligned}
$$

The derivations have to be done with respect to $b_{j}$ in addition to those with respect to $a_{i}$ :

$$
\begin{gathered}
\frac{\partial}{\partial a_{i}} E\left(\varepsilon^{2}(X, \dot{X}, Z)\right)=0 ; i=1 . . . k \text { and } \\
\frac{\partial}{\partial b_{j}} E\left(\varepsilon^{2}(X, \dot{X}, Z)\right)=0 ; j=1 \ldots . l .
\end{gathered}
$$

It has to be mentioned that the derivatives with respect to $b_{j}$ are more complicated then those with respect to $a_{i}$. The latter can be written as

$$
\begin{aligned}
\frac{\partial E\left[\varepsilon^{2}\right]}{\partial a_{i}}=E\left[2\left(\sum_{m=1}^{k} m a_{m} H^{m-1} \dot{X}+\sum_{m=1}^{l} b_{m} \operatorname{sgn}(X)|X|^{m}-c \dot{X}-k_{x} X-k_{z} Z\right) i H^{i-1} \dot{X}\right]=0 \\
i=1 \ldots k
\end{aligned}
$$

that can be rewritten as

$$
\begin{aligned}
& \sum_{m=1}^{k} m a_{m} E\left[H^{m+i-2} \dot{X}^{2}\right]+ \\
& \sum_{m 0=1}^{l} b_{m} E\left[\operatorname{sgn}(X)|X|^{m} H^{i-1} \dot{X}\right]= \\
& \quad c E\left[H^{i-1} \dot{X}^{2}\right]+k_{x} E\left[H^{i-1} \dot{X} X\right]+k_{z} E\left[H^{i-1} \dot{X} Z\right]
\end{aligned}
$$

While the former can be written as:

$$
\begin{aligned}
& \frac{\partial E\left[\varepsilon^{2}\right]}{\partial b_{j}}=E\left[2 \left(\sum_{m=1}^{k} m a_{m} H^{m-1} \dot{X}+\right.\right. \\
& \left.\sum_{m=1}^{l} b_{m} \operatorname{sgn}(X)|X|^{m}-c \dot{X}-k_{x} X-k_{z} Z\right) \\
& \left.\left(\sum_{m=1}^{k} m(m-1) a_{m} H^{m-2} \dot{X} \frac{1}{J+1}|X|^{j+1}+\operatorname{sgn}(\dot{X})\left|X^{j}\right|\right)\right] \\
& =0, \mathrm{j}=1 \ldots 1
\end{aligned}
$$

that becomes:

$$
\begin{aligned}
& \sum_{m=1}^{k} \sum_{n=1}^{k} \frac{1}{j+1} m(m-1) n a_{m} a_{n} E\left[H^{m+n-3}|H|^{j+1} \dot{X}^{2}\right]+ \\
& +\sum_{m=1}^{k} \sum_{n=1}^{l} \frac{1}{j+1} m(m-1) a_{m} b_{n} E \\
& {\left[H^{m-2} \operatorname{sgn}(X|X|)^{n+j+1} \dot{X}\right]+}
\end{aligned}
$$

$$
\begin{aligned}
& -\sum_{m=1}^{k} \frac{1}{j+1} m(m-1) a_{m} c E\left[H^{m-2}|X|^{j+1} \dot{X}^{2}\right]- \\
& \sum_{m=1}^{k} \frac{1}{j+1} m(m-1) a_{m} k_{x} E\left[H^{m-2} \operatorname{sgn}(X)|X|^{j+2} \dot{X}\right]+ \\
& -\sum_{m=1}^{k} \frac{1}{j+1} m(m-1) a_{m} k_{z} E\left[H^{m-2}|X|^{j+1} \dot{X} Z\right]+ \\
& \sum_{m=1}^{k} m a_{m} E\left[H^{m-1} \operatorname{sgn}(X)|X|^{j} \dot{X}\right]+ \\
& \sum_{m=1}^{i} b_{m} E\left[|X|^{m+j}\right]+-c E\left[\operatorname{sgn}(X)|X|^{j} \dot{X}\right]- \\
& k_{x} E\left[|X|^{j+1}\right]-k_{z} E\left[\operatorname{sgn}(X)|X|^{j} Z\right]=0
\end{aligned}
$$

The nonlinear restoring force $Z$ can be again estimated after the optimization assuming the error tends to zero by

$$
Z=\frac{1}{k_{z}}\left\{\begin{array}{l}
{\left[\sum_{i=1}^{k} i a_{i}\left(\frac{1}{2} \dot{X}^{2}+\sum_{j=1}^{l} \frac{b_{j}}{v+1}|X|^{j+1}\right)\right]} \\
\dot{X}+\sum_{j=1}^{l} b_{j}|X|^{j} \operatorname{sgn}(X)-c \dot{X}-k_{z} X
\end{array}\right\} .
$$

In this model the linear terms can be equalized by $a_{l}=c$ and $b_{1}=k_{x}$, this has as effect that the linear parts of both equations are identical. The energy will now become:

$$
H=\frac{1}{2} \dot{X}^{2}+\frac{1}{2} k_{x} X^{2}+\sum_{j=2}^{l} \frac{b_{j}}{j+1}|X|^{j+1},
$$

and the function $Q(H)$, that is needed to calculate the joint probability distribution, expands to

$$
\begin{aligned}
Q(H)= & c\left(\frac{1}{2} \dot{X}^{2}+\sum_{j=1}^{l} \frac{b_{j}}{j+1}|X|^{j+1}\right)+ \\
& \sum_{i=2}^{k} a_{i} \dot{X}^{2}\left(\frac{1}{2} \dot{X}^{2}+\sum_{j=1}^{l} \frac{b_{j}}{j+1}|X|^{j+1}\right)^{i} .
\end{aligned}
$$

The error function reduces to

$$
\begin{aligned}
\varepsilon= & {\left[\sum_{i=2}^{k} i a_{i}\left(\frac{1}{2} X^{2}+\frac{1}{2} k_{x} \dot{X}^{2}+\sum_{j=2}^{l} \frac{b_{j}}{j+1}|X|^{j+1}\right)^{i-1}\right], } \\
& \dot{X}+\sum_{j=2}^{l} b_{j}|X|^{j} \operatorname{sgn}(X)-k_{z} Z
\end{aligned}
$$

and of the hysteretic state variable $Z$ reduces to 


$$
Z=\frac{1}{k_{z}}\left\{\begin{array}{l}
\left.\left[\sum_{i=2}^{k} i a_{i}\left(\frac{1}{2} X^{2}+\frac{1}{2} k_{x} \dot{X}^{2}+\sum_{j=2}^{l} \frac{b_{j}}{v+1}|X|^{j+1}\right)^{i-1}\right]\right\} \\
\dot{X}+\sum_{j=2}^{l} b_{j}|X|^{j} \operatorname{sgn}(X)
\end{array}\right\}
$$

Of course the derivation is now only needed for the remaining variables $a_{2}$ up to $a_{k}$ and $b_{2}$ up to $b_{l}$.

Another problem that arises if method 2 is chosen, because one has to derive the moments from the potential model, is due to the computational effort necessary for solving the two dimensional numerical integrals. Nevertheless, Eqs.(22-25), that simplify the calculation of these integrals, can be simply extended if the following nonlinear restoring force is chosen:

$$
r(X)=b \operatorname{sgn}(X)|X|^{v} \text {. }
$$

In this case only one additional integral has to be solved numerically. Eq.(23) becomes

$$
E\left[H^{i} \dot{X}^{2}\right]=\alpha E\left[H^{i+1}\right] \text {, }
$$

And Eq.(25) has to be changed in

$$
E\left[H^{i+k-1}\right]=\frac{1}{k a_{k}}\left\{\begin{array}{l}
\left(\frac{1}{\alpha}+i-1\right) \pi S_{0} E\left[H^{i-1}\right] \\
-\sum_{j=1}^{k-1} j a_{j} E\left[H^{i+j-1}\right]
\end{array}\right\},
$$

$i=1, k-1$.

At least the derivatives of the error estimate reduces to

$$
\begin{aligned}
& \frac{\partial E\left[\varepsilon^{2}\right]}{\partial a_{i}}=E\left[2\left(\sum_{m=1}^{k} m a_{m} H^{m-1} X+b \operatorname{sgn}(X)\right) i H^{i} \dot{X}\right]=0, \\
& i=1, k-1 .
\end{aligned}
$$

and

$$
\begin{aligned}
& \frac{\partial E\left[\varepsilon^{2}\right]}{\partial b}=E\left[2\left(\begin{array}{c}
\sum_{m=1}^{k} m a_{m} H^{m-1} \dot{X}+ \\
b \operatorname{sgn}(H)|X|^{v}-c X-k_{x} \dot{X}-k_{z Z}
\end{array}\right)\right. \\
& \left.\left(\sum_{m=1}^{k} m(m-1) a_{m} H^{m-2} \dot{X} \frac{1}{v+1}\left|X^{v+1}\right|+\operatorname{sgn}(X)|X|^{v}\right)\right] . \\
& =0 \quad \mathrm{j}=1 \ldots \mathrm{i}
\end{aligned}
$$

\section{GENERALIZATION FOR DIFFERENT LOAD INTENSITIES}

As mentioned before, it is not the main purpose of this study to present a non linearization method, but to use this method as a tool to approximate the behavior of a given hysteresis by a potential model. The non linearization discussed earlier refers to one specific load level. Therefore, the response of the equivalent model will be only worth for this level. Especially in the observed case, the Bouc model, with the chosen parameters, keeps always within a range of minus one to one. This will be not the case for the potential system, since the hysteretic behavior is directly connected to the displacement $\mathrm{X}$ and its time derivative.

In order to receive a global approximation the non linearization has been set in dependency of the load intensity $\mathrm{S}_{0 .}$. At least the parameters have to be evaluated at different load levels and an interpolated function will be established.

Due to some instabilities of the method 2 that are coming up, because of the chosen integration procedure of the semi infinite integral, if the parameter $a_{3}$ becomes negative, during the iteration, the method 1 will be used to present the dependency of the parameters in accordance of the intensity of the load

$$
K K=2 \pi S_{0}
$$

Fig. (13) displays the behavior of the displacement $X$ with respect to time $t$ of the Bouc model and of the approximating potential system. Fig. (14) presents the dependency of the parameters $a_{i}$ of the potential system on the load intensity $K K$. It is obvious that there is only a slight change of the parameters due to the change of the load intensity. This is of great importance, because the approximation of a measured hysteretic behavior of a material has not to be performed at great amount of different load levels. This reduces the effort rapidly. In a calculation the parameters can be kept constant, if the load level varies within a bounded domain. This means also, that the potential models seem to be globally applicable to hysteretic behavior.

When the load intensity increases, the values of the coefficients of the higher order terms of the potential series (namely $a_{2}$ and $a_{3}$ ) tend to zero, this means that the distribution of the state variables tends to become Gaussian (see Eq. (12)). It is also visible that with greater load intensities the approximation becomes better (Fig. 15). This might be astonishing, since the hysteretic behavior is of greater importance, but it was shown that the chosen approximation is not able to represent the cyclic behavior in an exact way. If the cycles become larger also the volume increases and therefore the cycles of the potential model will fit the cycles of the Bouc model in a better way.

Figs. (15 and 16) present this behavior at two extreme load intensities $K K=10$ and $K K=0.1$.

This problem can be solved, if $b_{j}$ are introduced as additional parameters as before mentioned. If the parameters are adjusted to measured data the equivalent non linearization is not appropriate. Therefore, the problems, that arise if this method is used, are of no importance for the case that the parameters are adjusted to measured hysteresis loops.

\section{CONCLUSION}

The possibility to use potential models for the approximation of the hysteretic behavior given by the Bouc model has been presented. The advantage is that the statistics of the response of the potential model are known in exact form, while the statistics of the Bouc system can be known 

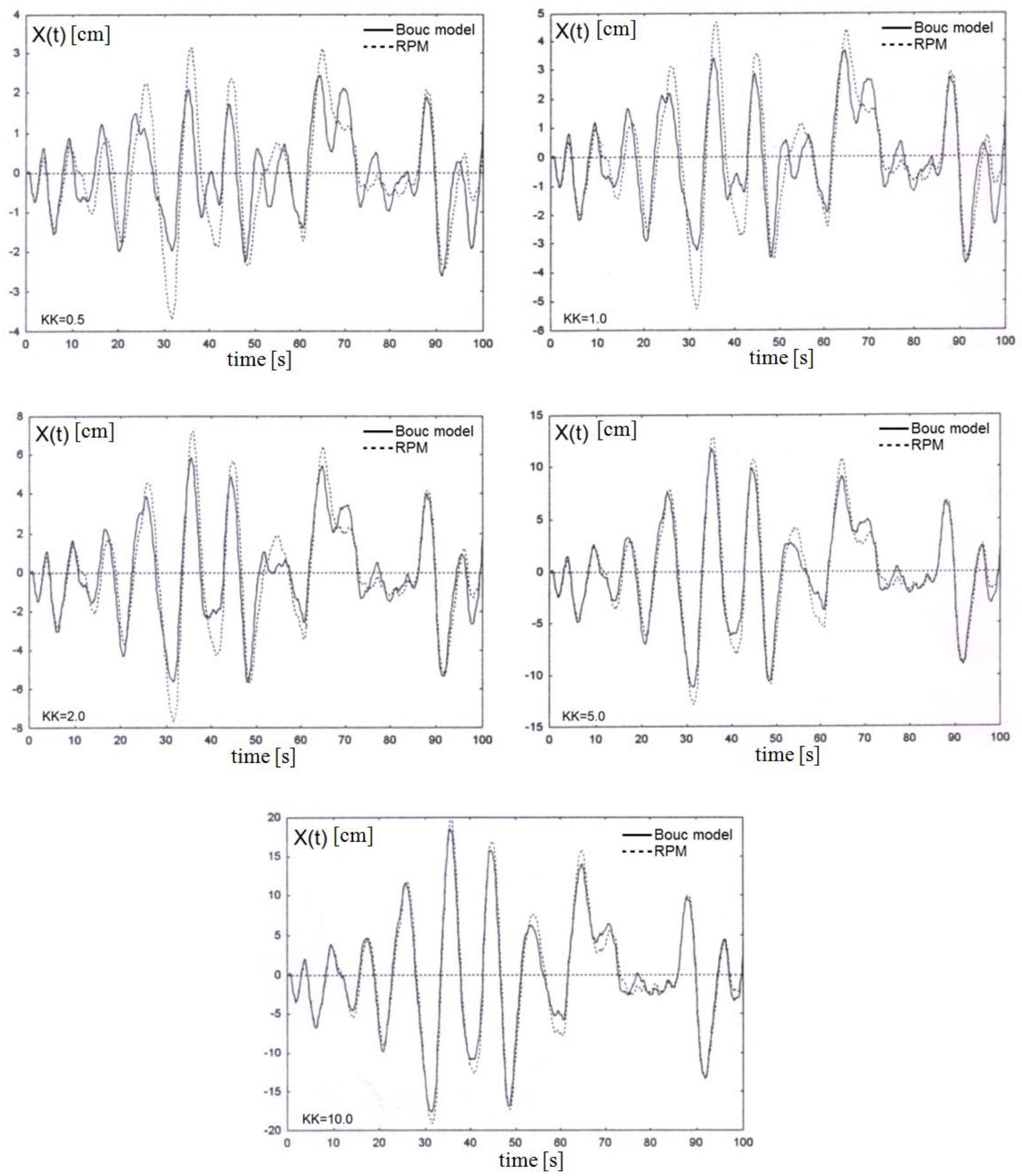

Fig. (13). Approximation of the Bouc model by a potential model $(\mathrm{k}=3)$ for different load intensities $\mathrm{KK}$ [ $\left.\mathrm{cm}^{2} / \mathrm{sec}^{3}\right]$.

only by a Monte Carlo simulation. Once the parameters defining an equivalent potential model are obtained they can be directly used to approximate the observed hysteretic behavior of materials. The strategy for obtaining the above parameters is a statistical equivalent non linearization that can include the dissipative forces or also the restoring forces. In the first case the non linearization can be used to determine regions for these parameters, which represent with good approximation the measured hysteretic behavior of tested materials. Analyses have shown that the better approximation in terms of shape of the displacement- hysteretic force cycles is obtained for great level of the external force. In order to obtain optimal results in the case of low level of the external forces the usage of a non linearization method to approximate the Bouc model including nonlinear restoring forces should be preferred, against the assumption of a linear restoring force in the potential model equal to the linear restoring force in the Bouc model. 


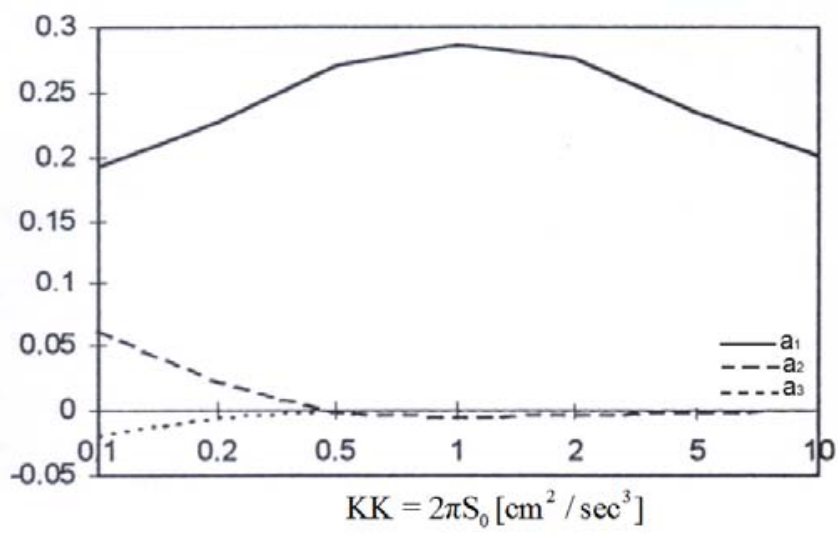

Fig. (14). Parameters $a_{1}$ to $a_{3}$ in accordance of the load intensity $K K=2 \pi S_{0}$.
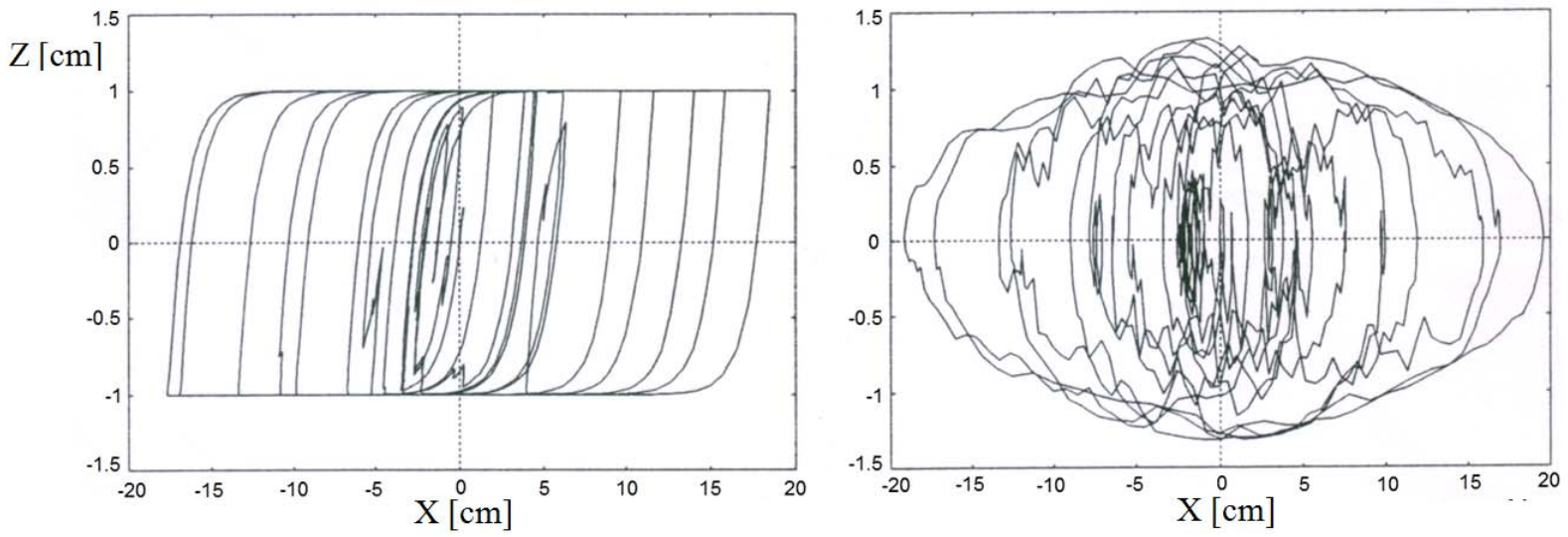

Fig. (15). Hysteretic cycles in X-Z plane for the Bouc model and equivalent potential model $\mathrm{KK}=10 \mathrm{~cm}^{2} / \mathrm{sec}^{3}$.
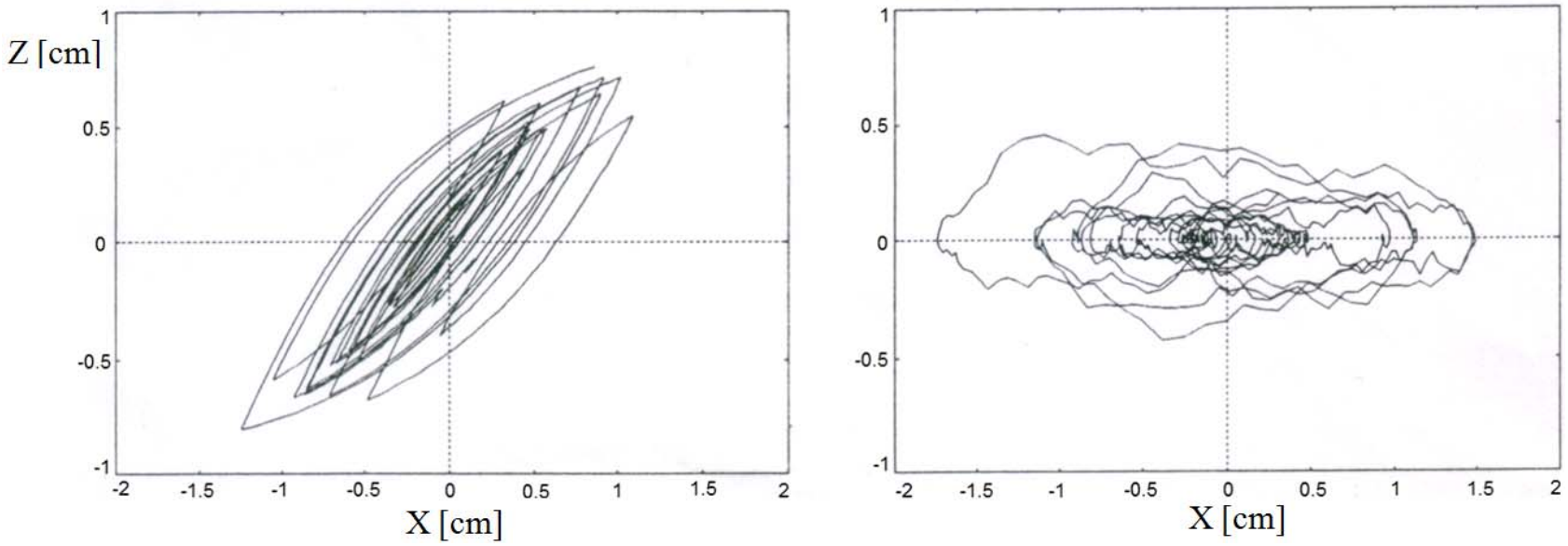

Fig. (16). Hysteretic cycles in $\mathrm{X}-\mathrm{Z}$ plane for the Bouc model and equivalent potential model $\mathrm{KK}=0.1 \mathrm{~cm}^{2} / \mathrm{sec}^{3}$.

However it must be noted that nonlinear restoring forces in the potential model show an increase in the computational effort for the estimation of the equivalent parameters with respect to obtain faster result when the non linearization involves only the nonlinear dissipating forces.

\section{CONFLICT OF INTEREST}

The authors confirm that this article content has no conflict of interest.

\section{ACKNOWLEDGEMENTS}

Declared none.

\section{REFERENCES}

[1] R. Bouc, "Forced vibration of a mechanical systems with hysteresis", In: Proceedings of the Fourth Conference on Nonlinear Oscillations, Prague, Czechoslovakia, 1967, p. 315.

[2] T. Barber, and M.N. Noori, "Modeling general hysteresis behavior and random vibration application", J. Eng. Mech., ASCE, vol. 107, pp. 1069-89, 1986. 
[3] Y. Suzuki, and R. Minai, "Application of stochastic differential equations to seismic reliability analysis of hysteretic structures", Prob. Eng. Mech., vol. 3, pp. 43-52, 1988.

[4] H. Grunndamann, and H. Waubke, "Non linear stochastic dynamics of system with random properties: A spectral approach combined with statistical linearization", Int. J. Non-Linear Mech, vol. 31, pp. 619-30, 1996.

[5] H. Jalali, "An alternative linearization approach to hysteretic system", Communi Nonlinear Sci Numer Simul., vol. 19, pp. 245-57, 2014.

[6] W-Q. Zhu, and J-S. Yu, "The equivalent non-linear system method", J. Sound Vib., vol. 129, pp. 385-95, 1989.

[7] L. Cavaleri, and M. Di Paola, "Statistic moments of the total energy of potential systems and applications to equivalent nonlinearization", Int. J. Non-Linear Mech., vol. 35, pp. 573-87, 2000.

[8] L. Cavaleri, M. Di Paola and G. Failla, "Some properties of multidegree of freedom potential systems and application to statistical equivalent non-linearization", Int. J. Non-Linear Mech., vol. 38, pp. 405-21, 2003.

[9] H.S. Al-Nimry, "Quasi static testing of RC infilled frames and confined stone-concrete bearing walls", J. Earthquake Eng., vol. 18, pp. 1-23, 2014.

[10] L. Cavaleri, M. Papia, and M. Fossetti, "Infilled frames: developments in the evaluation of the cyclic behaviour under lateral loads", Struc. Eng. Mech., vol. 21, pp. 469-94, 2005.
[11] R. Ceravolo, S. Erlicher, and L. Zanotti-Fragonara, " Comparison of restoring force models for the identification of structures with hysteretic and degradation", J. Sound Vib., vol. 332, pp. 6982-99, 2013.

[12] L. Cavaleri, and M. Papia, "A new dynamic identification technique: application to the evaluation of the equivalent strut for infilled frames", Eng. Struct., vol. 25, pp. 889-901, 2003.

[13] L. Cavaleri, "Identification of stiffness dissipation and input parameters of randomly excited non-linear systems: capability of Restricted Potential Models (RPM)", Int. J. Non-Linear Mech., vol. 41, pp. 1068-83, 2006.

[14] S. Benfratello, L. Cavaleri, and M. Papia, "Identification of stiffness, dissipation and input parameters of multi degree of freedom civil systems under unmeasured base excitations", Probab. Eng. Mech., vol. 24, pp. 190-8, 2009.

[15] L. Cavaleri, M. Di Paola, and G. Failla, "Risposta approssimata di sistemi isteretici soggetti a forzanti aleatorie", In: Proceedings of the Ninth Conference "L'Ingegneria Sismica in Italia". Torino, Italy, 1999, pp. 20-23.

[16] L. Cavaleri, and M. Papia, "An output-only stochastic parametric approach for the identification of linear and nonlinear structures under random base excitations: Advances and comparisons", Prob. Eng. Mech., vol. 35, pp. 11-21, 2014.

Received: February 26, 2014

Revised: May 27, 2014

Accepted: May 27, 2014

(C) Cavaleri et al.; Licensee Bentham Open.

This is an open access article licensed under the terms of the Creative Commons Attribution Non-Commercial License (http://creativecommons.org/licenses/by-nc/3.0/) which permits unrestricted, non-commercial use, distribution and reproduction in any medium, provided the work is properly cited. 